\title{
Both obesity-prone and obesity-resistant rats present delayed cutaneous wound healing
}

\author{
Adriana Paulino do Nascimento and Andréa Monte-Alto-Costa* \\ Tissue Repair Laboratory, Histology and Embryology Department, State University of Rio de Janeiro, Rua Professor Manuel de \\ Abreu, 444, $3^{\circ}$ Andar, 20550-170 Rio de Janeiro, RJ, Brazil
}

(Received 20 April 2010 - Revised 4 July 2010 - Accepted 20 January 2011 - First published online 9 May 2011)

\section{Abstract}

Diet-induced overweight rats exhibit delayed cutaneous healing; however, when receiving an obesogenic diet, some rats are susceptible to developing the overweight phenotype, whereas others are resistant. We investigated cutaneous healing in diet-induced obesity (DIO)prone and diet-resistant (DR) rats. Male rats were fed with a standard (control) or a high-saturated fat (30\% fat, w/w) diet for 20 weeks. Then, the experimental group was subdivided into DIO ( $n$ 17) and DR ( $n$ 16) groups. An excision lesion was made, and the animals were killed 7 or $14 \mathrm{~d}$ later. The average body weight was 29 and $25 \%$ higher in the DIO group compared with the C and DR groups. Retroperitoneal fat was higher in the DIO group than in the control and DR groups (518 and 92\%) and was higher in the DR group than in the control group (223\%). The DIO group presented glucose intolerance, and both the DIO and DR groups presented delayed wound contraction (50\%) and re-epithelialisation (20\%). Compared with the DR group, the DIO group displayed higher amounts of inflammatory cells as well as higher levels of lipid peroxidation $(P<0.05)$. Myofibroblastic differentiation and vessel remodelling were delayed in both the DIO and DR groups. Nitrite levels were lower in the DIO group (340\% less) than in the DR group. TNF- $\alpha$ expression was increased in the DIO group (130\%) compared with the DR group. Our results showed that DIO as well as DR rats present delays in cutaneous wound healing, even though the DR group does not have an overweight phenotype.

Key words: Cutaneous wound healing: Obesogenic diet: Obesity-prone rats: Obesity-resistant rats

The loss of integrity over large areas of skin as a result of injury or illness may lead to major disability or even death. The primary goals of wound treatments are rapid wound closure and a functionally and aesthetically satisfactory scar ${ }^{(1)}$. Wound healing is a complicated and highly regulated series of cellular and biochemical events involving input from, and the interplay between, many cell types. Reactive oxygen species are regulators of this process; they are required for protection against invading pathogens ${ }^{(1)}$, and low levels of reactive oxygen species are also essential mediators of intracellular signalling ${ }^{(2)}$. The process of wound healing is divided into three phases: inflammation; granulation tissue formation; remodelling ${ }^{(3)}$. Cytokine synthesis is necessary for wounds to heal; however, excessive amounts of inflammatory cytokines may disturb the process (as observed in chronic non-healing wounds such as ulcers $)^{(4,5)}$. Various products of white adipose tissue (WAT) are linked specifically to inflammation ${ }^{(6)}$, suggesting the participation of these factors in the transition from acute to chronic wounds.
A prolonged healing process represents a complication that contributes to morbidity and mortality. Obesity is a condition in which adipose tissue excess is associated with chronic lowgrade inflammation, indicated by leucocytosis ${ }^{(7)}$, increased plasma levels of inflammatory cytokines ${ }^{(8,9)}$ and increased abundance of macrophages in adipose tissue ${ }^{(10)}$. Overweight and obesity are problems of epidemic proportions among humans and are associated with an increased incidence of many persistent conditions, such as chronic wounds ${ }^{(11)}$. Data from a previous study in our laboratory ${ }^{(12)}$ showed that an overweight phenotype induced by a high-fat (HF) diet, without correlation with comorbidity or the metabolic syndrome, delayed the cutaneous wound healing process through elongation of the inflammatory phase.

The ideal model to study adipose tissue increase is the one that mimics the symptoms observed in human subjects; rodents fed a HF diet are an excellent model of obesity in which the dietary environment is a major contributor ${ }^{(13)}$. Human subjects and other mammals show considerable

Abbreviations: 4-HNE, 4-hydroxynonenal-positive cell; DIO, diet-induced obesity; DR, diet resistant; HF, high fat; PMN, polymorphonuclear; Vv, volume density; WAT, white adipose tissue.

*Corresponding author: Dr A. Monte-Alto-Costa, fax +55 212587 6511, email amacosta@uerj.br 
inter-individual variation in susceptibility to weight gain in response to obesogenic diets. Rats fed a HF diet exhibit a bimodal pattern in body-weight gain similar to that observed in human subjects. Approximately, half of the rats gain weight rapidly compared with standard chow-fed rats and develop diet-induced obesity (DIO), whereas the other half gain body weight at a rate similar to or lower than that of standard chow-fed rats and are thus characterised as diet-resistant $(\mathrm{DR})^{(14)}$. This model has been of great interest because the genesis of obesity proneness and obesity resistance within the same rat strain without genetic or surgical modification is similar to that observed in human subjects and enables one to dissociate between the factors related to diet and obesity per se.

Although it is known that overweight induced by a HF diet delays cutaneous wound healing, the pattern of wound healing in individuals who eat an obesogenic diet but are resistant to developing obesity is not known. We hypothesise that the consumption of an obesogenic diet, without inducing overweight, can affect cutaneous wound healing; thus the aim of the present study was to evaluate the cutaneous wound healing of DIO-prone and DR rats 7 and $14 \mathrm{~d}$ after wounding.

\section{Materials and methods}

\section{Experimental animals}

The present study was approved by the Ethical Committee for Animal Use of the State University of Rio de Janeiro (CEA/160/ 2006). After weaning, male Wistar rats weighing between 30 and $60 \mathrm{~g}$ started diet protocols with free access to food and water throughout the experimental period. Animals were kept in a room with controlled temperature $\left(22^{\circ} \mathrm{C}\right)$ on a $12 \mathrm{~h}$ light $-12 \mathrm{~h}$ dark cycle. The rats were divided into three groups of at least seven animals each and were studied at two different time points (fifty-seven rats began the experiment; fifty rats were included in the study and seven rats were excluded from the study as explained later).

\section{Diet protocol}

The rats were randomly divided into control $(n 17)$ and diet $(n$ 40) groups. The control group was fed with commercial pellets $(23 \%$ protein, $71 \%$ carbohydrate and $6 \%$ fat (percentage of dietary weight), $18 \mathrm{~kJ} / \mathrm{g}$; Nuvilab ${ }^{\circledR}$; Nuvital, Colombo, PR, Brazil), and the diet group was fed with a high-saturated fat diet known to induce obesity in susceptible animals (15\% protein, $55 \%$ carbohydrate, $30 \%$ fat (percentage of dietary weight), $23 \mathrm{~kJ} / \mathrm{g}$, about $45 \%$ of energy intake as fat $)^{(12,15)}$. The source of fat was shortening, a semi-solid fat composed of hydrogenated soyabean oil that contains 30\% trans-fatty acids (Primor ${ }^{\circledR}$; Bunge Alimentos S. A., Florianópolis, SC, Brazil). Food intake was measured daily, and body weight was recorded weekly. Rats on the obesogenic diet were separated on the basis of body-weight gain at the end of week 20; rats with a high weight gain were designated as DIO rats ( $n$ 17, body-weight gain $\geq 350 \mathrm{~g}$ ), and rats with a low weight gain were designated as DR rats ( $n$ 16, body-weight gain $\leq 300 \mathrm{~g}$ ). The remaining rats $(n 7)$ were excluded from the study because they were between the two limits of weight gain (Fig. 1). The DIO and DR rats remained on the obesogenic diet until the end of the experiment.

\section{Wounding model and macroscopic analyses}

After 20 weeks of the diet (day 0), the rats were intraperitoneally anaesthetised with ketamine $(5 \mathrm{mg} / \mathrm{kg})$ and xylazine ( $2 \mathrm{mg} / \mathrm{kg}$ ). After shaving the dorsum, a full-thickness excision wound $\left(1 \mathrm{~cm}^{2}\right)$ was made to the level of the panniculus carnosus. The wound was not sutured or covered and healed by second intention. The discomfort induced by this wound protocol was minimal, so no analgesics were required. To evaluate wound contraction and re-epithelialisation, a transparent plastic sheet was placed over each wound and its margins were traced ${ }^{(12,16)}$. The wound area was measured and photographed soon after wounding, and weekly thereafter until euthanasia, without scab removal. Re-epithelialisation was measured 7 and $14 \mathrm{~d}$ after wounding. After digitisation, the wound area was measured using KS400 image software (Zeiss-Vision, Oberkochen, Germany). Data are expressed as a percentage of the initial wound area and as a percentage of the re-epithelialised wound area.

\section{Oral glucose tolerance test}

At the end of 20 weeks of diet, following a $12-14 \mathrm{~h}$ fast but before wounding, glucose concentration from the tail tip was determined (time 0) in six randomly selected animals per group, and then each rat received an oral administration of glucose $(2 \mathrm{~g} / \mathrm{kg})$. Blood samples were collected from the tail tip at 15, 30, 60 and 120 min after the glucose challenge ${ }^{(17)}$. Glucose concentration in the blood was assessed using a reagent strip (Accu-Chek Advantage; Roche Diagnostics, Mannheim, Germany). Results of the oral glucose tolerance test are expressed as integrated areas under the curves (AUC) over 120 min for glucose calculated using the trapezoid rule ${ }^{(18)}$ :

$$
\mathrm{AUC}=\left(C_{1}+C_{2}\right)(2)^{-1} \times\left(t_{2}-t_{1}\right),
$$

where $C_{1}$ and $C_{2}$ are blood glucose at times $0\left(t_{1}\right)$ and $15 \mathrm{~min}$ $\left(t_{2}\right)$, respectively, after the glucose load.

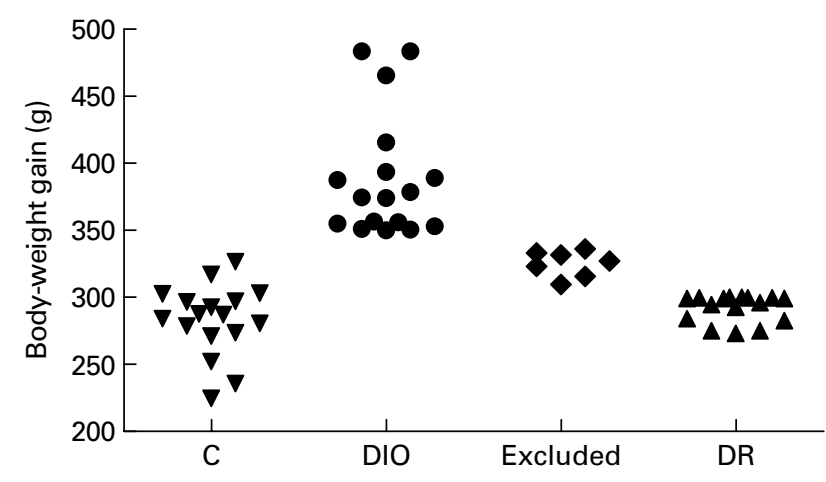

Fig. 1. Distribution of body-weight gain of all animals at the end of 20 weeks of ad libitum access to food. The control (C), diet-induced obese (DIO) and diet-resistant (DR) groups were composed of seventeen, seventeen and sixteen animals, respectively, and seven animals were excluded from the experiment. 


\section{Tissue harvesting and microscopic analyses}

Rats were killed by $\mathrm{CO}_{2}$ exposure $7 \mathrm{~d}$ (control, $n 7$; DIO, $n 8$; DR, $n$ 7) or $14 \mathrm{~d}$ (control, $n$ 10; DIO, $n$ 9; DR, $n$ 9) after wounding. A fragment of the lesion and adjacent normal skin was formalin-fixed and paraffin-embedded, and another fragment of the lesion was frozen $\left(-70^{\circ} \mathrm{C}\right)$. WAT from the retroperitoneal pad was carefully removed, weighed and frozen $\left(-70^{\circ} \mathrm{C}\right)$.

Paraffin sections were stained with haematoxylin-eosin to quantify the number of polymorphonuclear (PMN) leucocytes in the granulation tissue; ten random fields $\left(0.02 \mathrm{~mm}^{2}\right)$ per animal were analysed using a $100 \times$ objective (Zeiss Primo Star; Zeiss-Vision). Sections were also stained with toluidine blue to evaluate the number of mast cells; between six and ten random fields per animal $\left(0.15 \mathrm{~mm}^{2}\right)$ were analysed in the deep region of the granulation tissue using a $40 \times$ objective (Zeiss Primo Star; Zeiss-Vision). Results are expressed as cells $/ \mathrm{mm}^{2}$, and the quantifications were performed blindly and repeated without significant differences between them.

Additionally, sections were stained with Sirius red and observed under polarisation to evaluate the organisation of collagen fibres. Thick collagen fibres, characteristic of tissues with high densities of collagen, appeared strongly birefringent and yellow to red in colour, whereas thin collagen fibres, indicative of tissues with a low density of collagen fibres, were weakly birefringent and greenish.

\section{Immunohistochemistry and quantifications}

For the quantification of CD68-positive macrophages, 4-hydroxynonenal-positive cells (4-HNE), myofibroblasts and blood vessels, sections were incubated with mouse anti-CD68 (1:500; Serotec, Inc., Raleigh, NC, USA), goat anti-4-HNE (1:4000; Santa Cruz Biotechnology, Inc., Santa Cruz, CA, USA) and mouse anti- $\alpha$-smooth muscle actin (1:1000; DAKO, Carpinteria, CA, USA), respectively. For antigen retrieval, the sections were digested with $0 \cdot 1 \%$ trypsin (Difco Laboratories, Detroit, MI, USA) before labelling. Subsequently, the sections were incubated with $3 \% \mathrm{H}_{2} \mathrm{O}_{2}$ in methanol to inhibit endogenous peroxidase activity and then incubated with the primary antibody. After washing, the primary antibodies anti-CD68 and anti- $\alpha$-smooth muscle actin were detected using the EnVision system ${ }^{\circledR}$ (DAKO), and anti-4-HNE was detected using a biotinylated donkey anti-goat IgG antibody (Santa Cruz Biotechnology, Inc.) and streptavidin-horseradish peroxidase (DAKO). Diaminobenzidine was used as a chromogen. The sections were counterstained with haematoxylin. The negative controls on sections, where the primary antibodies were omitted, exhibited no labelling.

To quantify CD68-positive macrophages, fifteen random fields $\left(3636 \mu \mathrm{m}^{2}\right)$ per animal were analysed in the granulation tissue using a $40 \times$ objective. For this, a video microscope system (Axiostar Plus Zeiss microscope (Zeiss, Oberkochen, Germany), Pixelink video camera (PixeLINK, Ottawa, Canada) and Intelbras monitor (Intelbras, Santa Catarina, Brazil)) was used. Data are presented as the average of CD68-positive macrophages $/ \mathrm{mm}^{2}$. To quantify the number of 4-HNE-positive cells, as 4-HNE is a major product of endogenous lipid peroxidation $^{(19)}$, fifteen random fields $\left(0.02 \mathrm{~mm}^{2}\right)$ per animal were analysed using a $100 \times$ objective (Zeiss Primo Star; ZeissVision). Results are expressed as cells $/ \mathrm{mm}^{2}$.

Because stereological methods are precise tools that allow for the acquisition of information about three-dimensional structures based on observations made in two-dimensional sections ${ }^{(20,21)}$, the quantitative distribution of myofibroblasts and blood vessels (expressing $\alpha$-smooth muscle actin) was evaluated using this method (point counting) ${ }^{(12)}$. Vessels were identified by the presence of blood cells within the lumens or positive staining for $\alpha$-smooth muscle actin in the wall (to confirm the presence of smooth muscle cells or pericytes) ${ }^{(12)}$. For this quantification, eight random fields per animal were analysed using a $40 \times$ objective for myofibroblasts, and five random fields per animal were analysed in both the superficial and deep regions of granulation tissue using a $20 \times$ objective for blood vessels. For analyses, a video microscope system (Axiostar Plus Zeiss microscope,

Table 1. Body weight (BW), daily food intake, retroperitoneal fat pad, wound contraction and re-epithelialisation in the control (C), diet-induced obesity (DIO) and diet-resistant (DR) groups*

(Mean values with their standard errors of at least seven animals per group)

\begin{tabular}{|c|c|c|c|c|c|c|}
\hline & \multicolumn{2}{|c|}{$\mathrm{C}$} & \multicolumn{2}{|c|}{ DIO } & \multicolumn{2}{|c|}{ DR } \\
\hline & Mean & SEM & Mean & SEM & Mean & SEM \\
\hline \multicolumn{7}{|l|}{ Parameter } \\
\hline Initial BW (g) & $47 \cdot 5$ & $2 \cdot 3$ & $45 \cdot 5$ & 1.3 & $38 \cdot 7$ & 2.6 \\
\hline Final BW (g) & $323.4^{\mathrm{a}}$ & 8.7 & $414 \cdot 4^{\mathrm{b}}$ & $16 \cdot 7$ & $332 \cdot 3^{\mathrm{a}}$ & 7.5 \\
\hline Food consumption $(\mathrm{g} / \mathrm{d})$ & $18 \cdot 8^{\mathrm{a}, \mathrm{b}}$ & 0.6 & $19 \cdot 7^{\mathrm{a}}$ & 1.1 & $17 \cdot 1^{\mathrm{b}}$ & 0.9 \\
\hline Food consumption $(\mathrm{kJ} / \mathrm{d})$ & $339 \cdot 1^{\mathrm{a}}$ & 11.4 & $453 \cdot 6^{\mathrm{b}}$ & $25 \cdot 1$ & $393.4^{\mathrm{c}}$ & $19 \cdot 8$ \\
\hline Retroperitoneal fat $(\mathrm{g})$ & $2 \cdot 2^{\mathrm{a}}$ & 0.5 & $13 \cdot 6^{\mathrm{b}}$ & 1.4 & $7 \cdot 1^{\mathrm{c}}$ & 0.5 \\
\hline Retroperitoneal fat (\% final BW) & $0.7^{\mathrm{a}}$ & 0.1 & $3 \cdot 2^{\mathrm{b}}$ & 0.2 & $2 \cdot 1^{\mathrm{c}}$ & 0.1 \\
\hline Wound area after day 7 (\% initial) & $57 \cdot 3^{\mathrm{a}}$ & 1.6 & $88 \cdot 8^{\mathrm{b}}$ & 1.8 & $86 \cdot 3^{\mathrm{b}}$ & $2 \cdot 7$ \\
\hline Wound area after day 14 (\% initial) & $26 \cdot 0$ & $1 \cdot 0$ & 28.7 & $1 \cdot 1$ & $26 \cdot 7$ & 0.8 \\
\hline Re-epithelialised wound area after day 7 (\%) & 3.6 & 1.9 & 0.0 & 0.0 & 1.4 & 1.0 \\
\hline Re-epithelialised wound area after day $14(\%)$ & $70 \cdot 0^{\mathrm{a}}$ & 2.7 & $57.4^{\mathrm{b}}$ & 3.7 & $55 \cdot 8^{\mathrm{b}}$ & 3.6 \\
\hline
\end{tabular}

Day 7, $7 \mathrm{~d}$ after wounding; day 14, $14 \mathrm{~d}$ after wounding.

a,b,c Mean values within a row with unlike superscript letters were significantly different (one-way ANOVA with Bonferroni's post-test; $P<0.05$ ).

* Body weight, daily food intake, retroperitoneal fat pad $(C=17, \mathrm{DIO}=17, \mathrm{DR}=16)$; wound contraction and re-epithelialisation after day 7

$(C=7, \mathrm{DIO}=8, \mathrm{DR}=7)$; wound contraction and re-epithelialisation after day $14(C=10, \mathrm{DIO}=9, \mathrm{DR}=9)$. 
Pixelink video camera and Intelbras monitor) was used. Data are expressed as volume density ( $\mathrm{Vv}$ ) of myofibroblasts (Vv(myofibroblasts)\%) and blood vessels (Vv(blood vessels) \%) in the superficial and deep regions.

All quantifications were performed blindly and were reproduced without significant differences.

\section{Biochemical analyses}

Collagen deposition was quantified by the hydroxyproline assay in frozen lesions ${ }^{(22)}$. Dry and defatted tissue (3.5$11.0 \mathrm{mg}$ ) was hydrolysed in $6 \mathrm{M}-\mathrm{HCl}$ for $18 \mathrm{~h}$ at $118^{\circ} \mathrm{C}$. Hydrolysate was then diluted with distilled water, neutralised with $6 \mathrm{~m}-\mathrm{NaOH}$ and centrifuged at $3000 \mathrm{rpm}$ for $15 \mathrm{~min}$. Hydroxyproline levels were measured in this hydrolysate as described previously ${ }^{(23)}$. Briefly, aliquots of hydrolysate $(80 \mu \mathrm{l})$ were mixed with $40 \mu \mathrm{l}$ chloramine-T (0.05 M; Merck \& Company, Inc., Whitehouse Station, NJ, USA) and incubated for $20 \mathrm{~min}$ at $25^{\circ} \mathrm{C}$. Then, $40 \mu \mathrm{l}$ perchloric acid $(3.17 \mathrm{M}$; Vetec, Rio de Janeiro, Brazil) and $40 \mu \mathrm{l}$ 4-dimethylamino benzaldehyde (Merck \& Company, Inc.) were added and incubated for $20 \mathrm{~min}$ at $60^{\circ} \mathrm{C}$. The developed colour was read spectrophotometrically at $550 \mathrm{~nm}$. Hydroxyproline concentrations were determined from a standard curve generated by different concentrations of L-4-hydroxyproline (Sigma-Aldrich, St Louis, MO, USA). Data are expressed as ng hydroxyproline/mg tissue.

Levels of nitrite in wound lysates were determined by a spectrophotometric method based on the Griess reaction ${ }^{(24)}$. The detection limit for nitrite using this method is 1.0$5.0 \mu \mathrm{m}$. An aliquot of each sample $(100 \mu \mathrm{l})$ was mixed and incubated with $100 \mu \mathrm{l}$ of Griess reagent (1\% sulphanilamide in $5 \%$ phosphoric acid and $0 \cdot 1 \%$ naphthalene diamine dihydrochloride in water) at room temperature for $10 \mathrm{~min}$. The developed colour was then read spectrophotometrically at $550 \mathrm{~nm}$. Nitrite concentrations in the samples were determined from a standard curve generated by different concentrations of sodium nitrite $(0 \cdot 1-100 \mu \mathrm{M})$. Data are expressed as $\mu \mathrm{mol}$ nitrite $/ \mu \mathrm{g}$ total protein.

All analyses were performed in triplicate and were reproduced without significant differences.

\section{ELISA}

Frozen retroperitoneal fat tissue fragments $(0.5 \mathrm{~g})$ were homogenised in $1 \mathrm{ml}$ of PBS solution containing $0.5 \%$ Tween 20 and protease inhibitors. Retroperitoneal fat samples were subjected to ELISA analyses for TNF- $\alpha$ (PeproTech, Rocky Hill, NJ, USA) according to the manufacturer's instructions. The TNF- $\alpha$ ELISA development kit contains key components required for the quantitative measurement of TNF- $\alpha$ within the range of $32-2000 \mathrm{pg} / \mathrm{ml}$. The samples were quantified using a standard curve $(0-2 \mathrm{ng} / \mathrm{ml})$. Data are expressed as pg of TNF- $\alpha / \mathrm{g}$ of retroperitoneal fat tissue.

\section{Statistical analyses}

Data were analysed using a one-way ANOVA with Bonferroni's post-test (body mass, retroperitoneal fat, wound contraction, re-epithelialisation and blood glucose levels), unpaired $t$ test (nitrite and hydroxyproline levels, and ELISA) or Mann-Whitney test for data that were not normally distributed (inflammatory cell and 4-HNE-positive cell quantifications and stereological analyses) using the software GraphPad InStat version 3.01 (GraphPad Software, Inc., San Diego, CA, USA). Values of $P<0.05$ were considered statistically significant. The results are shown as means with their standard errors.

\section{Results}

\section{Body weight and retroperitoneal fat-pad weight}

The initial mean body weight of the control, DIO and DR groups did not differ (Table 1). At week 6, the DIO group (219.4 (SEM 6.3) g) presented a higher average body weight than the DR group (188.5 (SEM 6.6) g; $P<0.01$, data not shown), and the body weight remained higher throughout the experimental period (Table 1). At week 11, the body weight of the DIO group $(347.5$ (SEM 14.7) g) was greater than that of the control group $(306.5$ (SEM 3.7) g; $P<0.01$, data not shown) and remained higher until the end of the experiment (Table 1). On the day the lesion was performed (week 20, day 0), the body weight was 28.8 and $24.6 \%$ higher in the DIO group compared with the control and DR
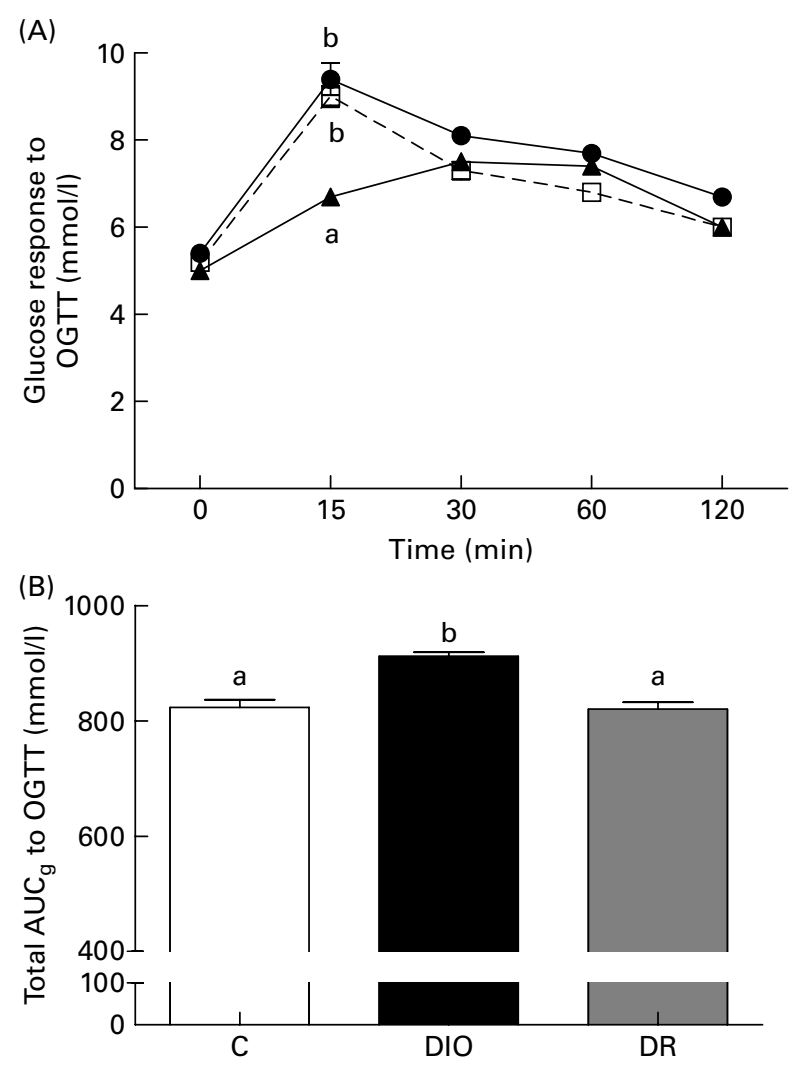

Fig. 2. (A) Fasting glucose and blood glucose response to the oral glucose tolerance test (OGTT), $(B)$ total area under curve glucose $\left(A U C_{g}\right)$ to the OGTT after 20 weeks of diet in the control $\left(C,-\square_{--}\right)$, diet-induced obese (DIO, - - ) and diet-resistant (DR, - - ) rats. Values are means of six randomly selected animals per group, with their standard errors represented by vertical bars. ${ }^{\mathrm{a}, \mathrm{b}}$ Mean values of groups with unlike letters were significantly different (one-way ANOVA with Bonferroni's post-test; $P<0.05$ ). 
Table 2. Microscopic and biochemical analyses of wounded areas 7 and $14 \mathrm{~d}$ after wounding*

(Mean values with their standard errors of at least seven animals per group for microscopic analyses and of six animals per group randomly selected for biochemical analyses)

\begin{tabular}{|c|c|c|c|c|}
\hline & \multicolumn{2}{|c|}{ DIO } & \multicolumn{2}{|c|}{ DR } \\
\hline & Mean & SEM & Mean & SEM \\
\hline \multicolumn{5}{|l|}{ Measurements } \\
\hline PMN leucocytes $/ \mathrm{mm}^{2}$ (day 7 ) & $531^{\mathrm{a}}$ & 0.9 & $398^{\mathrm{b}}$ & 1.0 \\
\hline PMN leucocytes $/ \mathrm{mm}^{2}$ (day 14) & 161 & 0.4 & 167 & 0.5 \\
\hline Mast cells $/ \mathrm{mm}^{2}$ (day 7 ) & $43^{a}$ & 0.6 & $27^{\mathrm{b}}$ & 0.5 \\
\hline Mast cells $/ \mathrm{mm}^{2}$ (day 14) & $24^{a}$ & 0.5 & $17^{\mathrm{b}}$ & 0.5 \\
\hline CD68-postitive macrophages $/ \mathrm{mm}^{2}$ (day 7) & 2478 & 115 & 2627 & 111 \\
\hline CD68-postitive macrophages $/ \mathrm{mm}^{2}$ (day 14) & $2542^{\mathrm{a}}$ & 105 & $2235^{\mathrm{b}}$ & 121 \\
\hline 4-HNE-postitive cells $/ \mathrm{mm}^{2}$ (day 7) & $234^{\mathrm{a}}$ & 0.4 & $184^{\mathrm{b}}$ & 0.3 \\
\hline 4-HNE-postitive cells $/ \mathrm{mm}^{2}$ (day 14 ) & 284 & 0.4 & 270 & 0.4 \\
\hline Vv (myofibroblasts) \% (day 7) & 6.5 & $0 \cdot 8$ & $4 \cdot 8$ & 0.7 \\
\hline Vv (myofibroblasts) \% (day 14) & $17 \cdot 4^{\mathrm{a}}$ & $1 \cdot 2$ & $10 \cdot 8^{\mathrm{b}}$ & 1.0 \\
\hline Vv (blood vessels) \% (day 7) & $8 \cdot 3$ & $0 \cdot 8$ & $7 \cdot 6$ & 0.7 \\
\hline Vv (blood vessels) \% (day 14) & $8 \cdot 1$ & 0.9 & $8 \cdot 3$ & 0.9 \\
\hline Hydroxyproline (ng/mg tissue) (day 7) & 0.4 & 0.06 & 0.4 & 0.02 \\
\hline Hydroxyproline (ng/mg tissue) (day 14) & 0.5 & 0.04 & 0.6 & 0.05 \\
\hline Nitrite ( $\mu \mathrm{mol} / \mu \mathrm{g}$ protein) (day 7 ) & 34.1 & $6 \cdot 8$ & 34.0 & $9 \cdot 1$ \\
\hline Nitrite $(\mu \mathrm{mol} / \mu \mathrm{g}$ protein) (day 14$)$ & $2 \cdot 0^{\mathrm{a}}$ & 0.3 & $8 \cdot 8^{\mathrm{b}}$ & 2.5 \\
\hline
\end{tabular}

DIO, diet-induced obese group; DR, diet-resistant group; PMN, polymorphonuclear; day 7, 7d after wounding; day 14, $14 \mathrm{~d}$ after wounding; HNE, 4-hydroxynonenal-positive cells; $\mathrm{Vv}$, volume density.

${ }^{a, b}$ Mean values within a row with unlike superscript letters were significantly different (Mann-Whitney test for microscopic analyses and unpaired $t$ test for biochemical analyses; $P<0.05$ ).

* Microscopic analyses after day $7: \mathrm{DIO}=8, \mathrm{DR}=7$; microscopic analyses after day 14: DIO =9, DR =9; biochemical analyses after days 7 and 14 : $\mathrm{DIO}=6, \mathrm{DR}=6$.

groups, respectively. The DIO group consumed more food than the DR group; however, there was no difference in the food intake between the control and obesogenic diet (DIO and DR) groups (Table 1). Furthermore, the DIO group consumed significantly more energy per day than the control and DR groups throughout the study (Table 1), and the DR group consumed significantly more energy per day than the control group (Table 1).

As shown in Table 1, retroperitoneal fat mass was higher in the DIO group than in the control and DR groups (518.2 and $91.5 \%$, respectively, $P<0.05)$. The retroperitoneal fat mass was higher in the DR group than in the control group $(222.7 \%, \quad P<0.05)$. The retroperitoneal fat percentage showed the same pattern of results (Table 1).

\section{Fasting glucose and oral glucose tolerance test}

There was no difference between the average fasting blood glucose of the groups (Fig. 2(A)). The blood glucose level of the DR group was lower than the glucose levels of the control and DIO groups $(P<0.05$; Fig. 2(A) 15 min after the oral glucose tolerance test. After the glucose load, blood glucose levels over a period of $120 \mathrm{~min}$ in the DIO group were higher than in the control and DR groups. The glucose area under curve was significantly higher in the DIO group compared with the control and DR groups $(P<0.05$; Fig. 2(B)).

\section{Macroscopic analyses}

The wound area was greater in the DIO $(55.0 \%, P<0.05)$ and DR $(50.6 \%, P<0.05)$ groups than in the control group $7 \mathrm{~d}$ after wounding (Table 1). However, there was no difference in the area between the groups $14 \mathrm{~d}$ after wounding (Table 1).

The DIO group showed no sign of re-epithelialisation $7 \mathrm{~d}$ after wounding, whereas the control and DR groups presented re-epithelialisation at a low rate. The percentage of the re-epithelialised wound area was lower in the DIO $(-18.0 \%$, $P<0.05)$ and DR $(-20.3 \%, P<0.05)$ groups compared with the control group $14 \mathrm{~d}$ after wounding (Table 1 ).

\section{Quantification of inflammatory infiltrate}

The inflammatory infiltrate was intense in the DIO and DR groups $7 \mathrm{~d}$ after wounding. The number of PMN leucocytes was greater in the $\operatorname{DIO}(33.4 \%, P<0.05)$ group than in the DR group (Table 2). In both groups, mast cells were localised in the deep region, but this was especially prominent in the DIO group, where the mast cells were near the adipocytes (Fig. 3(A)). The number of mast cells was greater in the DIO group (59.3\%, $P<0.05)$ than in the DR group (Table 2; Fig. 3((A) and (B))). The number of CD68-positive macrophages was similar in both groups (Table 2).

The DIO group still had a high inflammatory infiltrate $14 \mathrm{~d}$ after wounding. The number of PMN leucocytes decreased in both groups, with no differences between them (Table 2). The number of mast cells remained higher in the DIO group than in the DR group $(41.2 \%, P<0.05)$ (Table 2$)$. In addition, the number of CD68-positive macrophages decreased slightly in the DR group but remained elevated in the DIO group. In the DIO group, the CD68-positive macrophages were localised around adipocytes, in close association with them (Fig. 3(C) inset). The number of CD68-positive macrophages was higher in the DIO group than in the DR group $(13.7 \%$, $P<0 \cdot 05$; Table 2 and Fig. 3(C) and (D))). 

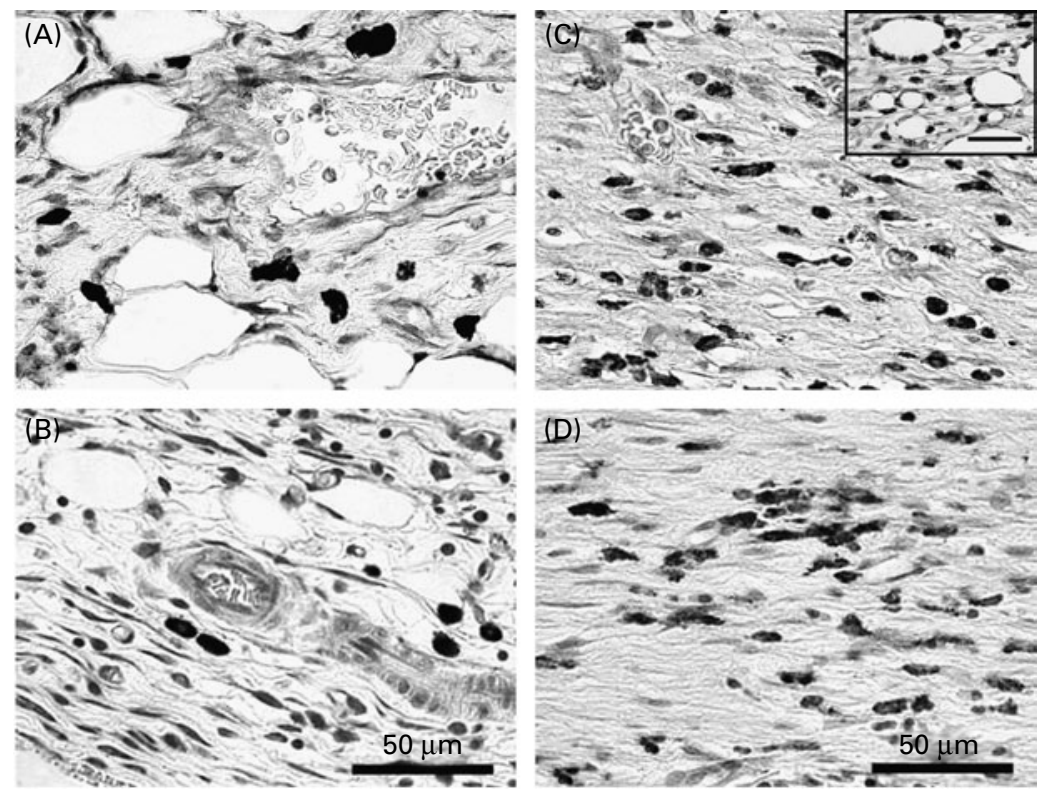

Fig. 3. (A, B) Distribution of mast cells and (C, D) CD68-positive macrophages 7 and $14 \mathrm{~d}$ after wounding. The diet-induced obese (DIO (A, C)) and diet-resistant (DR (B, D)) groups are shown. Mast cells were analysed in toluidine blue-stained sections, and macrophages were identified by immunohistochemistry against CD68 in sections. To evaluate the number of mast cells, between six and ten random fields per animal were analysed (at least seven animals per group). To quantify CD68-positive macrophages, fifteen random fields per animal were analysed (at least seven animals per group). All quantifications were performed blindly and repeated. Scale bar $=50 \mu \mathrm{m}$. Mobilisation of mast cells was higher in (A) the DIO group than in (B) the DR group. The amount of CD68-positive macrophages was higher in (C) the DIO group than in (D) the DR group. In detail (C), CD68-positive macrophages around adipocytes in the DIO group can be observed. Microscopic analyses after day $7(\mathrm{DIO}=8, \mathrm{DR}=7)$; microscopic analyses after day $14(\mathrm{DIO}=9, \mathrm{DR}=9)$.

\section{Lipid peroxidation}

To evaluate the tissue damage due to the inflammatory response in the DIO and DR rats, we assayed the 4-HNE levels in wound sections. 4-HNE is formed during lipid peroxidation and is present in higher levels during oxidative stress ${ }^{(19)}$. The number of 4-HNE-positive cells was higher in the DIO group than in the DR group $(27.2 \%, P<0.05) 7 \mathrm{~d}$ after wounding (Table 2). There was no difference between the DIO and DR groups $14 \mathrm{~d}$ after wounding

\section{Development of granulation tissue and angiogenesis}

Myofibroblasts were equally scarce in the DIO and DR groups $7 \mathrm{~d}$ after wounding (Table 2). However, $14 \mathrm{~d}$ after wounding, this pattern was reversed, with myofibroblasts found in all granulation tissues of the DIO and DR groups. The Vv of myofibroblasts was greater in the DIO group than in the DR group (61.1\%,P<0.05; Table 2$)$.

There was no difference in the volume occupied by blood vessels between groups at all time points in the superficial and deep regions of the wound. Furthermore, the Vv of blood vessels $14 \mathrm{~d}$ after wounding remained high in the DIO and DR groups compared with the $\mathrm{Vv} 7 \mathrm{~d}$ after wounding (Table 2).

\section{Collagen deposition}

In both groups, $7 \mathrm{~d}$ after wounding, most of the collagen fibres were yellow-greenish and a few were yellow-reddish, and the yellow-reddish were short and had a random arrangement. However, $14 \mathrm{~d}$ after wounding, most collagen fibres were yellow-reddish, elongated and arranged parallel to the surface. To confirm these histological observations, hydroxyproline levels were measured. In the DIO and DR groups, hydroxyproline levels were lower $7 \mathrm{~d}$ after wounding and increased slightly $14 \mathrm{~d}$ after wounding (Table 2 ). There was no difference in the levels between the groups.

\section{Measurement of nitrite levels}

Because nitrite is a stable molecule and accounts for more than $90 \%$ of total measurable nitrite and nitrate ${ }^{(25)}$, the nitrite dosage was used as an index of NO synthesis. There was no difference between the groups $7 \mathrm{~d}$ after wounding; however, $14 \mathrm{~d}$ after wounding, the nitrite levels were lower in the DIO group $(-340 \%)$ than in the DR group $(P<0.05$; Table 2$)$.

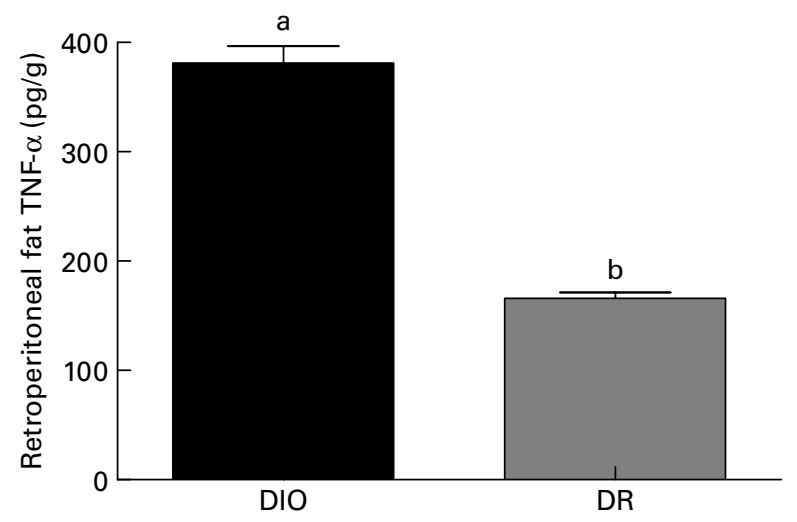

Fig. 4. TNF- $\alpha$ levels in the retroperitoneal fat of the diet-induced obese (DIO) and diet-resistant (DR) rats. Values are means of six randomly selected animals per group, with their standard errors represented by vertical bars. ${ }^{a, b}$ Mean values of a group with unlike letters were significantly different (unpaired $t$ test; $P<0.05$ ). 


\section{Measurement of $T N F-\alpha$ release}

Within the same group, no differences were observed in the TNF- $\alpha$ production from animals killed 7 or $14 \mathrm{~d}$ after wounding (data not shown). As shown in Fig. 4 , the TNF- $\alpha$ amount in retroperitoneal fat was higher in the DIO group than in the DR group $(130 \%, P<0.05)$ at both 7 and $14 \mathrm{~d}$ after wounding.

\section{Discussion}

Overweight induced by a HF diet delays cutaneous wound healing in obesity-prone rats through the elongation of the inflammatory phase ${ }^{(12)}$. Nevertheless, the pattern of wound healing of individuals who are resistant to an increase in body weight when fed an obesogenic diet is unknown. In the present study, both DIO and DR rats presented delayed wound healing; however, DIO rats had a more intense inflammatory phase and more oxidative damage.

Overweight and obesity are problems of epidemic proportions and are associated with an increased incidence of many chronic conditions, such as chronic wounds ${ }^{(11)}$. In rodents, an obesogenic diet promotes obesity through hyperphagia ${ }^{(26)}$; this finding was corroborated by the results of the present study. The DIO group ingested more food and showed a higher body weight than the control and DR groups, although the latter was fed the same diet. Importantly, obesogenic DIO occurs in some but not all rats ${ }^{(14)}$. In the present study, the weight of retroperitoneal fat mass was higher in the DIO group, lower in the control group and intermediate in the DR group, indicating that not only hyperphagia, but also the fat contained in the diet, plays an important role in the increase in adipose tissue. This finding is confirmed by the fact that the control and DR groups consumed equivalent amounts of food, yet the DR group had a higher amount of retroperitoneal fat mass than the control group. According to the literature, various products of WAT are specifically linked to inflammation ${ }^{(6)}$, and the inflammatory phase is disturbed during the cutaneous wound healing of obesity-prone rats $^{(12)}$. However, wound healing (and inflammatory phase duration) has never been studied in obesity-resistant rats.

Although obesity-induced chronic inflammation is related to insulin resistance and the development of type 2 diabetes $^{(27)}$, no difference in fasting blood glucose was observed in the present study. Previous studies with Wistar rats fed a HF diet showed no metabolically significant hyperglycaemia ${ }^{(28)}$. A mild fasting hyperglycaemia with a long-term (approximately 10 months) HF feed has been observed ${ }^{(29)}$; however, our diet protocol lasted only 20 weeks. Although the obesogenic diet did not cause an elevation in fasting glucose, the DIO group presented a small degree of glucose intolerance; the area under the glucose level curve was significantly elevated in the DIO group compared with the control and DR groups, indicating a possible early stage of insulin resistance development. Borst \& Conover ${ }^{(28)}$ reported that the induction of an insulin-resistant state by HF feeding is accompanied by increased adiposity and increased tissue expression of TNF- $\alpha$ protein, supporting a possible autocrine/paracrine role for TNF- $\alpha$ in obesogenic diet-induced insulin resistance.
Importantly, glucose intolerance was only observed in the DIO group, which presented a substantial increase in retroperitoneal fat (sixfold) and of TNF- $\alpha$ protein in adipose tissue. However, the blood glucose concentration does not report all of the information regarding glucose metabolism, and further studies assessing the insulin concentration are required.

The resolution of the inflammatory response is a limiting factor in successful repair after tissue injury. Most non-healing wounds fail to progress through the normal phases of wound repair, remaining in a chronic inflammation state ${ }^{(4,5)}$. The inflammatory response is necessary, because it induces the production of pro-inflammatory cytokines, recruits macrophages and stimulates the proliferation of resident cells and angiogenesis ${ }^{(30)}$. In the present study, the DIO group presented a higher number of inflammatory cells and mast cells than the DR group. WAT from obese animals contain more mast cells than WAT from their lean counterparts, and these cells promote DIO and glucose intolerance ${ }^{(31)}$. This result indicates a more prominent inflammatory phase in the DIO group. A body of evidence suggests the presence of an overall, low-grade inflammation in obesity, with altered levels of several circulating factors, such as an increase in the plasma levels of C-reactive protein, TNF- $\alpha$, IL- 6 and other biological markers of inflammation ${ }^{(32-34)}$. This association is meaningful, and several animal models have suggested that these inflammatory processes have a causal relationship with obesity and its comorbidities. Thus, the increase in TNF- $\alpha$ in adipose tissue of the DIO rats may explain the large number of inflammatory cells in this group. Furthermore, there is a strong positive correlation between the degree of adiposity and several obesity-associated disorders ${ }^{(35)}$. This association was observed in the present study, as the DR group presented only an intermediate increase in retroperitoneal fat mass but did not develop obesity, did not increase levels of TNF- $\alpha$ and possessed a lower number of inflammatory cells than the DIO group. Although the DR group did not develop obesity, it showed delayed wound healing. A previous study has shown differential regulation of some inflammation-related genes in adipose tissue between the DIO and DR rats. DIO rats presented an increase in TNF- $\alpha$, IL- 6 and haptoglobin mRNA levels, whereas DR rats only presented an increase in IL-6 mRNA levels ${ }^{(36)}$, suggesting different regulation of the inflammatory healing phase. Regardless of these differences, the result is deleterious to wound repair. However, more studies are needed to define the inflammatory status of the DIO and DR rats during wound healing.

Reactive oxygen species are produced in response to cutaneous injury $^{(37)}$ and may cause cellular damage by peroxidation of membrane lipids, inactivation of sulfhydryl enzymes, cross-linking of proteins and breakdown of $\mathrm{DNA}^{(38)}$. In most studies, reactive oxygen species levels at the wound site have been determined indirectly through analyses of oxidation products of lipids, proteins or $\mathrm{DNA}^{(39)}$. A major product of lipid peroxidation, 4-HNE, can be detected by immunohistochemistry $^{(40)}$. In the present study, the amount of 4-HNEpositive cells was greater in the DIO group than in the DR group. The number of inflammatory cells was also greater in 
the DIO group and was probably responsible for the increased lipid peroxidation.

Wound healing involves a variety of processes such as acute inflammation, cell proliferation and wound contraction ${ }^{(1)}$. These phases overlap in time and duration, each depending on various factors such as tissue type, health status, nutritional status and the presence of infections. Several types of cells are recruited to an injury site to carry out the processes of repair. In rodents, wound contraction is the main mechanism of wound closure ${ }^{(41)}$, and myofibroblasts are the main cells responsible for wound contraction ${ }^{(42,43)}$. In the present study, the DIO and DR groups presented delayed wound contraction $7 \mathrm{~d}$ after wounding. This result can be explained by the delay in myofibroblastic differentiation observed at this time point in the groups. A lack of apoptotic neutrophils at the wound site deprives the macrophages of their main stimulus to secrete transforming growth factor- $\beta 1$, a key mediator involved in myofibroblast differentiation from fibroblasts ${ }^{(44,45)}$. Transforming growth factor- $\beta 1$ derived from macrophages also acts as a strong inhibitor of the inducible isoform of $\mathrm{NO}$ synthase during wound healing ${ }^{(46)}$. This association was corroborated by the present results; $7 \mathrm{~d}$ after wounding, the number of PMN leucocytes and nitrite levels was elevated in the DIO and DR groups. These results indicate that the large number of PMN leucocytes may be related to the delay in myofibroblastic differentiation and increased NO synthesis in the DIO and DR groups.

Re-epithelialisation is the process of restoring the epidermis and consists of proliferation and migration of keratinocytes ${ }^{(47)}$. In the present study, the DIO and DR groups presented delayed re-epithelialisation. Keratinocyte proliferation and migration occurs, while the levels of immune cells and proinflammatory cytokines decrease ${ }^{(48)}$, and the present results showed that the DIO and DR groups still presented a large number of macrophages $14 \mathrm{~d}$ after wounding. Although the proliferation is increased in overweight rats ${ }^{(12)}$, the re-epithelialisation is still delayed, suggesting that the late, but considerable, presence of macrophages in the wounds of the DIO and DR groups compromised keratinocyte migration, consequently delaying re-epithelialisation.

When the levels of immune cells and pro-inflammatory cytokines decrease, fibroblasts and endothelial cells start to migrate into the wound and proliferate to initiate the second phase of healing, granulation tissue formation. Fibroblasts are attracted to the wound edge, proliferate and are responsible for the synthesis, deposition and remodelling of the extracellular matrix ${ }^{(1)}$. In the granulation tissue, the fibroblasts differentiate into myofibroblasts, which are responsible for wound contraction and for the production of the extracellular matrix $^{(42,43)}$. Although we observed a delay in myofibroblastic differentiation and the development of inflammation in the DIO and DR groups, there was no difference in the deposition and organisation of collagen fibres. The delay in myofibroblastic differentiation could have been counteracted by the increased NO by increased NO synthesis (nitrite dosage) in the DIO and DR groups $7 \mathrm{~d}$ after wounding. Studies have demonstrated that $\mathrm{NO}$ is able to stimulate extracellular matrix (mainly collagen) synthesis and deposition, and the link between NO and collagen deposition has been described in vivo and in vitro ${ }^{(49,50)}$. Another study from our laboratory ${ }^{(16)}$ has shown an increase in collagen deposition due to topical NO donor application in both inflammatory and proliferative phases.

To support the new tissue with oxygen and nutrients, blood vessels sprout at the edge of the wound, and the granulation tissue is continuously replenished by new blood vessels. Once the wound is filled with new granulation tissue, angiogenesis ceases and many blood vessels disintegrate as a result of apoptosis $^{(1)}$. In the present study, there was no difference in the volume occupied by blood vessels 7 and $14 \mathrm{~d}$ after wounding. However, $14 \mathrm{~d}$ after wounding, the volume occupied by blood vessels remained high in both groups. The high occupied volume was probably due to high cellularity, which was still observed in granulation tissue $14 \mathrm{~d}$ after wounding, suggesting that these vessels do not undergo apoptosis due to the high nutritional need still required by granulation tissue.

In conclusion, the obesogenic diet induced an increase in body weight and retroperitoneal fat mass in the DIO rats, whereas only an intermediate increase in retroperitoneal fat mass was observed in the DR rats. Although it did not induce weight gain, the obesogenic diet compromised (delayed) the wound healing process in the DR animals, highlighting the importance of diet composition in the wound healing process. More studies using purified diets are needed to distinguish the effects of fat level from the effects of fat type and/or effects of dietary protein level and quality on cutaneous wound healing of the DIO-prone and DR rats.

\section{Acknowledgements}

The present study was partially supported by FAPERJ. A. P. d. $\mathrm{N}$. holds a postgraduate fellowship from FAPERJ. Neither of the authors has conflicts of interest with respect to the present study. The contributions of the authors were as follows: A. P. d. N. designed and conducted the experiment, and wrote the manuscript. A. M.-A.-C. designed the experiment, critically analysed the results and participated in the critical revision of the manuscript. The authors thank Claudia Benjamim and Ariane Brogliato for their help in performing the ELISA.

\section{References}

1. Singer AJ \& Clark RA (1999) Cutaneous wound healing. $N$ Engl J Med 341, 738-746.

2. D'Autreaux B \& Toledano MB (2007) ROS as signalling molecules: mechanisms that generate specificity in ROS homeostasis. Nat Rev Mol Cell Biol 8, 813-824.

3. Broughton G 2nd, Janis JE \& Attinger CE (2006) The basic science of wound healing. Plast Reconstr Surg 117, 12S-34S.

4. Cooney R, Iocono J, Maish G, et al. (1997) Tumor necrosis factor mediates impaired wound healing in chronic abdominal sepsis. J Trauma 42, 415-420.

5. Trengove NJ, Bielefeldt-Ohmann H \& Stacey MC (2000) Mitogenic activity and cytokine levels in non-healing and healing chronic leg ulcers. Wound Repair Regen 8, 13-25.

6. Tilg H \& Moschen AR (2006) Adipocytokines: mediators linking adipose tissue, inflammation and immunity. Nat Rev Immunol 6, 772-783. 
7. Perfetto F, Mancuso F \& Tarquini R (2002) Leukocytosis and hyperleptinemia in obesity: is there a link? Haematologica 87, ELT25.

8. Dandona P, Weinstock R, Thusu K, et al. (1998) Tumor necrosis factor-alpha in sera of obese patients: fall with weight loss. J Clin Endocrinol Metab 83, 2907-2910.

9. Yudkin JS, Kumari M, Humphries SE, et al. (2000) Inflammation, obesity, stress and coronary heart disease: is interleukin-6 the link? Atherosclerosis 148, 209-214.

10. Zeyda M \& Stulnig TM (2007) Adipose tissue macrophages. Immunol Lett 112, 61-67.

11. Wilson JA \& Clark JJ (2004) Obesity: impediment to postsurgical wound healing. Adv Skin Wound Care 17, 426-432.

12. Nascimento AP \& Costa AM (2006) Overweight induced by high-fat diet delays rat cutaneous wound healing. Br J Nutr 96, 1069-1077.

13. Bullo M, Casas-Agustench P, Amigo-Correig P, et al. (2007) Inflammation, obesity and comorbidities: the role of diet. Public Health Nutr 10, 1164-1172.

14. Lauterio TJ, Bond JP \& Ulman EA (1994) Development and characterization of a purified diet to identify obesity-susceptible and resistant rat populations. J Nutr 124, 2172-2178.

15. Aoyama T, Fukui K, Takamatsu K, et al. (2000) Soy protein isolate and its hydrolysate reduce body fat of dietary obese rats and genetically obese mice (yellow KK). Nutrition 16, 349-354.

16. Amadeu TP, Seabra AB, de Oliveira MG, et al. (2008) Nitric oxide donor improves healing if applied on inflammatory and proliferative phase. J Surg Res 149, 84-93.

17. Anwer T, Sharma M, Pillai KK, et al. (2008) Effect of Withania somnifera on insulin sensitivity in non-insulin-dependent diabetes mellitus rats. Basic Clin Pharmacol Toxicol 102, 498-503.

18. Bhatt PA \& Makwana D (2008) Comparative influence of propranolol and verapamil on glycemic control and histamine sensitivity associated with L-thyroxine-induced hyperthyroidism - an experimental study. Fundam Clin Pharmacol 22, $53-59$.

19. Esterbauer H, Schaur RJ \& Zollner H (1991) Chemistry and biochemistry of 4-hydroxynonenal, malonaldehyde and related aldehydes. Free Radic Biol Med 11, 81-128.

20. Baddeley AJ, Gundersen HJ \& Cruz-Orive LM (1986) Estimation of surface area from vertical sections. I Microsc 142, Pt 3, 259-276.

21. Gundersen HJ, Bendtsen TF, Korbo L, et al. (1988) Some new, simple and efficient stereological methods and their use in pathological research and diagnosis. APMIS 96, 379-394.

22. Souza BR, Santos JS \& Costa AM (2006) Blockade of beta1and beta2-adrenoceptors delays wound contraction and re-epithelialization in rats. Clin Exp Pharmacol Physiol 33, 421-430.

23. Woessner JF Jr (1961) The determination of hydroxyproline in tissue and protein samples containing small proportions of this imino acid. Arch Biochem Biophys 93, 440-447.

24. Green LC, Wagner DA, Glogowski J, et al. (1982) Analysis of nitrate, nitrite, and [15N]nitrate in biological fluids. Anal Biochem 126, 131-138.

25. Schaffer MR, Tantry U, Efron PA, et al. (1997) Diabetesimpaired healing and reduced wound nitric oxide synthesis: a possible pathophysiologic correlation. Surgery 121, 513-519.

26. Oscai LB, Brown MM \& Miller WC (1984) Effect of dietary fat on food intake, growth and body composition in rats. Growth 48, 415-424.

27. Kahn SE, Zinman B, Haffner SM, et al. (2006) Obesity is a major determinant of the association of $\mathrm{C}$-reactive protein levels and the metabolic syndrome in type 2 diabetes. Diabetes 55, 2357-2364.

28. Borst SE \& Conover CF (2005) High-fat diet induces increased tissue expression of TNF-alpha. Life Sci 77, 2156-2165.

29. Chalkley SM, Hettiarachchi M, Chisholm DJ, et al. (2002) Long-term high-fat feeding leads to severe insulin resistance but not diabetes in Wistar rats. Am J Physiol Endocrinol Metab 282, E1231-E1238.

30. Eming SA, Krieg T \& Davidson JM (2007) Inflammation in wound repair: molecular and cellular mechanisms. J Invest Dermatol 127, 514-525.

31. Liu J, Divoux A, Sun J, et al. (2009) Genetic deficiency and pharmacological stabilization of mast cells reduce dietinduced obesity and diabetes in mice. Nat Med 15, 940-945.

32. Das UN (2002) Obesity, metabolic syndrome X, and inflammation. Nutrition 18, 430-432.

33. Engstrom G, Hedblad B, Stavenow L, et al. (2003) Inflammation-sensitive plasma proteins are associated with future weight gain. Diabetes 52, 2097-2101.

34. Mosca L (2002) C-reactive protein - to screen or not to screen? N Engl J Med 347, 1615-1617.

35. Janssen I, Heymsfield SB, Allison DB, et al. (2002) Body mass index and waist circumference independently contribute to the prediction of nonabdominal, abdominal subcutaneous, and visceral fat. Am J Clin Nutr 75, 683-688.

36. Perez-Echarri N, Perez-Matute P, Marcos-Gomez B, et al. (2008) Differential inflammatory status in rats susceptible or resistant to diet-induced obesity: effects of EPA ethyl ester treatment. Eur J Nutr 47, 380-386.

37. Gupta A, Singh RL \& Raghubir R (2002) Antioxidant status during cutaneous wound healing in immunocompromised rats. Mol Cell Biochem 241, 1-7.

38. Russo A, Longo R \& Vanella A (2002) Antioxidant activity of propolis: role of caffeic acid phenethyl ester and galangin. Fitoterapia 73, Suppl. 1, S21-S29.

39. Urso ML \& Clarkson PM (2003) Oxidative stress, exercise, and antioxidant supplementation. Toxicology 189, 41-54.

40. Ojha N, Roy S, He G, et al. (2008) Assessment of wound-site redox environment and the significance of Rac2 in cutaneous healing. Free Radic Biol Med 44, 682-691.

41. Davidson JM (1998) Animal models for wound repair. Arch Dermatol Res 290, S1-S11.

42. Desmouliere A, Chaponnier C \& Gabbiani G (2005) Tissue repair, contraction, and the myofibroblast. Wound Repair Regen 13, 7-12.

43. Gabbiani G (2003) The myofibroblast in wound healing and fibrocontractive diseases. J Pathol 200, 500-503.

44. Peters T, Sindrilaru A, Hinz B, et al. (2005) Wound-healing defect of $\mathrm{CD} 18(-/-)$ mice due to a decrease in TGFbeta1 and myofibroblast differentiation. Embo $J \mathbf{2 4}$, 3400-3410.

45. Tomasek JJ, Gabbiani G, Hinz B, et al. (2002) Myofibroblasts and mechano-regulation of connective tissue remodelling. Nat Rev Mol Cell Biol 3, 349-363.

46. Vodovotz Y, Bogdan C, Paik J, et al. (1993) Mechanisms of suppression of macrophage nitric oxide release by transforming growth factor beta. J Exp Med 178, 605-613.

47. Li J, Chen J \& Kirsner R (2007) Pathophysiology of acute wound healing. Clin Dermatol 25, 9-18.

48. Gurtner GC, Werner S, Barrandon Y, et al. (2008) Wound repair and regeneration. Nature $\mathbf{4 5 3}, 314-321$.

49. Rizk M, Witte MB \& Barbul A (2004) Nitric oxide and wound healing. World J Surg 28, 301-306.

50. Schwentker A, Vodovotz Y, Weller R, et al. (2002) Nitric oxide and wound repair: role of cytokines? Nitric Oxide $\mathbf{7}$, $1-10$. 